\title{
Using Rugby MSF Broadcast for Time Division Multiplexing Synchronisation in a Housing Community Sensor Network
}

\author{
John Maloco, Séamus McLoone and Declan T. Delaney \\ Department of Electronic Engineering, \\ National University of Ireland Maynooth \\ Maynooth, Co. Kildare, IRELAND \\ E-mail: john.maloco; seamus.mcloone@eeng.nuim.ie
}

\begin{abstract}
This paper presents the design of a new approach to networking a housing community, through the use of a mesh sensor network that consists of both wireless infrastructural mesh nodes and wireless sensor nodes. One key issue with such networks is the development of a suitable Medium Access Control (MAC) protocol for the purpose of transferring data from the sensor nodes to the infrastructural nodes. Here, we use a MAC protocol based on Time Division Multiplexing (TDM) and propose the use of the Rugby MSF broadcast as a synchronisation signal. This novel technique is investigated and some preliminary analysis is outlined.
\end{abstract}

Keywords - Mesh Sensor Networks, Medium Access Control (MAC), Time Division Multiplexing (TDM), Rugby MSF Signal.

\section{INTRODUCTION}

Much research has been carried out in recent years in the area of wireless applications for the home and living area. Automating the living space through wireless control and sensors is on the threshold of becoming commonplace in many homes. Systems such as Zigbee [1], Bluetooth [2] and Z-Wave [3] are being deployed to meet the requirements of wireless home automation.

Recently, wireless applications such as remote meter reading and radio frequency identification (RFID) tags [4] for refuse bins have found their way out of the house and into the wider community. Our research looks at extending this process by investigating a suitable wireless sensor network for a housing community that provides many different services, including the aforementioned ones. For example, a child tracking system would be a useful inclusion.

Networks already exist between many houses in housing estates. At present this is mainly through TCP/IP which, in most cases, is an indirect link provided by Internet Service Providers (ISPs). Wireless links are also becoming more prevalent, with Wireless Local Area Networks (WLANs). These are based on the IEEE 802.11 [5] standards. Many of these WLANs have a gateway to the Internet provided by wireless broadband service providers. Those without an Internet gateway can still form wireless communities to share data, music and video, play network games and/or partake in intranet activities.

Part of the reason why such wireless activities have become so popular is cost and legislation. These wireless devices are low cost and widely available. Furthermore, there is no licence required to operate them. They operate in the licence exempt Industry, Science and Medical (ISM) frequency bands. Radio modules for these frequency bands are available from numerous manufacturers, some as a single-chip solution.

The focus of our research is to develop a novel housing estate mesh sensor network based on licence exempt, low power wireless technology, not in competition with 802.11 [5]. This system should be both affordable and beneficial to a housing community. It also has the potential of being useful to service providers of the estate.

Sensor networks are at the forefront of interest among the wireless research community. These networks are a collection of intelligent nodes equipped with sensors and radios. The nodes work together to accomplish tasks such as asset tracking and environmental monitoring.

Wireless sensor networks commonly have the following characteristics:

- Low power: Battery operated devices which do not require servicing for long periods of time (typically 1-3 years). 
- Transmit small amounts of data: To ensure long battery life the radio transmitter is switched on for the shortest possible time to send small amounts of data (1 to 10 bytes). Short data transmissions also frees-up more channel space for other nodes to transmit.

- Low cost: Ideally the node should be low cost enabling many nodes to be deployed without having a price deterrent.

- Easy to deploy: It should be possible to add and remove nodes simply by powering them on and off, thus deploying them in an ad-hoc fashion.

- Reliability: Sensor nodes are unattended for long periods of time. Therefore they are designed to be reliable. This reliability extends to both hardware and software and also to a method of ensuring that sensor data reaches its destination.

Typically, sensor node networks are deployed in an ad-hoc fashion, often without much planning considerations. Frequently these nodes must organise themselves into a mesh network [6]. Mesh networks are ones in which nodes transmit data to adjacent nodes. These nodes act as routers and forward the data on to its destination, either directly (single-hop) or, via additional routing nodes (multi-hop).

In mesh sensor networks the mesh node usually incorporates a hardwired sensor. In this paper, we employ a network where the sensors are wirelessly connected to the mesh nodes, thus allowing for greater flexibility in sensor deployment. One key issue with this type of network is the development of a suitable Medium Access Control (MAC) protocol [7] for the purpose of transferring data from the sensors to the mesh nodes. Here, we use a MAC protocol based on Time Division Multiplexing (TDM) and propose the use of the Rugby MSF broadcast as a synchronisation signal.

The rest of this paper is structured as follows. In the next section an overview of the mesh sensor network system design is presented. In Section III a Medium Access Control (MAC) method for the wireless sensor nodes is described. Section IV investigates the use of the Rugby MSF time signal as a synchronisation source for a Time Division Multiplexing (TDM) data protocol. Preliminary analysis and results are given in Section V. The paper ends with some conclusions and suggestions for future work.

\section{SYSTEM DESCRIPTION}

\section{a) System components}

Figure 1 below is a depiction of the proposed mesh sensor network. The system consists of a base station, wireless mesh nodes and wireless sensor nodes.

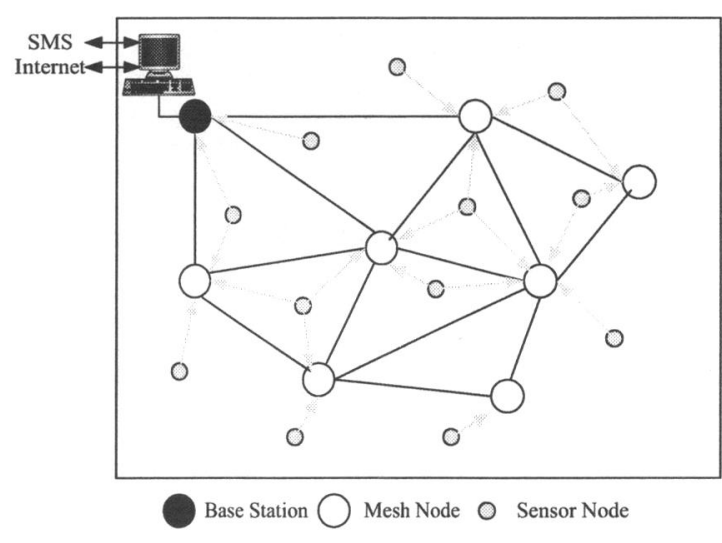

Figure 1: System Topology

Mesh Nodes: These nodes form a wireless mesh infrastructure, giving full coverage of the housing estate. They act as wireless routers in the system, relaying sensor data in a single and multi hop fashion. Additional nodes may be added and removed on an ad-hoc basis.

The mesh node contains a dedicated radio receiver to interface to the sensor node, operating at the $433 \mathrm{MHz}$ ISM band frequency. It also contains a transceiver operating at $868 \mathrm{MHz}$, used to interface to other mesh nodes in the network. A PIC microcontroller [8] provides the intelligence as shown in Figure 2. These nodes are powered continuously by a combination of rechargeable battery and solar panel. The sole purpose of these nodes is to collect data from the sensor nodes and pass this data to the Base Station in the most efficient way.

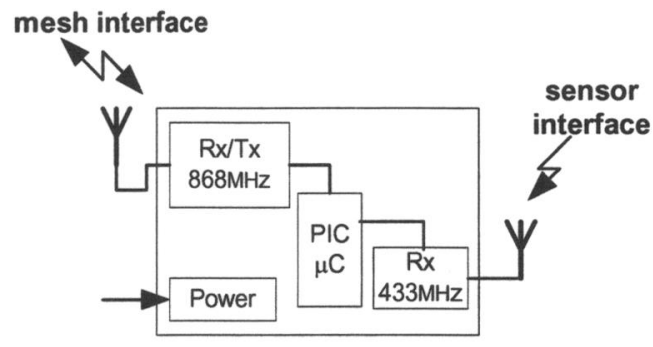

Figure 2: Mesh node

Sensor nodes: These are battery powered single channel wireless devices, see Figure 3. They are capable of sending data to any/all infrastructure mesh nodes within radio range. They have transmit-only capability for data. The main function of these devices is to read sensor inputs and transmit the results to a mesh node.

The sensor node contains a dedicated MSF radio receiver. It also consists of an rfPIC [8] which provides the intelligence and incorporates a radio transmitter, as shown in Figure 3. An external sensor interface is also included. 


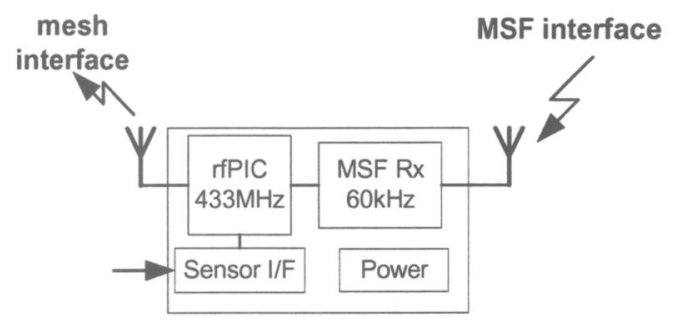

Figure 3: Sensor Node

Base Station: This configures and co-ordinates the mesh network. It consists of a base node and a PC. The PC provides the processing power of the base station. The base node is the interface between the mesh network and a PC. It contains a PIC microcontroller and a radio transceiver. The main purpose of the base station is to receive and process data from the sensor nodes. The base station provides the only external information access point to the network. It interfaces to the outside world via SMS and the Internet.

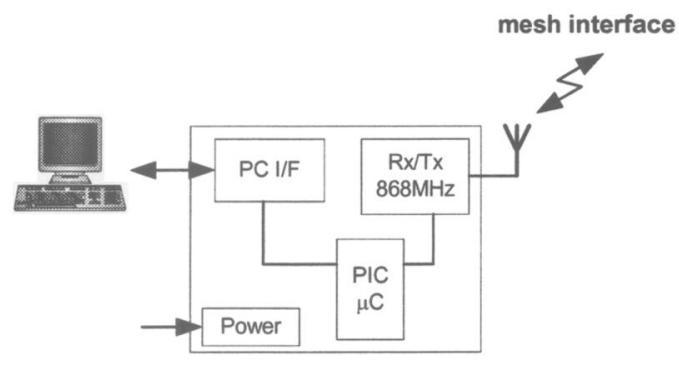

Figure 4: Base Station

\section{b) Physical Deployment}

The layout of housing estates is, in general, conducive to the network deployment. It is proposed to mount infrastructural mesh nodes on rooftops and high-up on lamp posts. This greatly reduces physical obstruction to the radio transmissions. In addition large open areas in housing estates have little or no electrical radio interference.

\section{c) Key Benefits}

The system has many possible uses including:

- Oil tank monitoring: Oil level sensors could be deployed to report low levels of home heating oil in tanks. This data is then relayed to the base station. The base station can then make this information available to the house owner via SMS or to an oil supplier over the internet.

- House alarm notification: A sensor node can be used to detect when a house alarm is activated. This information can then be passed to the house owner or to a nominated third party.

- Radiolocation: The system can be used for locating assets and people, for example the location monitoring of a child.
- Refuse services: Some refuse collection services already employ RFID tags to identify bins while they are being emptied. Having prior knowledge of weight, or whether a bin needs collecting, could help the service provider better manage the refuse collections.

- Personal aid notification: Some sensor nodes may be used to signal the need for personal aid, particularly in the case of the disabled and elderly.

It is worth noting that the proposed system is not intended to replace existing security or alarm systems. Instead it is intended for information and notification purposes.

\section{MAC PROTOCOL FOR SENSOR NODES}

The basic structure of the mesh sensor network was outlined in the previous section. Here, a suitable MAC protocol is investigated to enable the sensor nodes transfer their data successfully to the mesh nodes.

Two of the main design criteria for the sensor nodes are minimum power consumption, i.e. maximum battery life, and a reliable method for transferring data. Ideally the sensor nodes should operate as follows: from an initial powered down/sleep mode, they switch on, send a short data burst and switch off /sleep. This method can work if the system contains only a few nodes. However, with a large number of sensor nodes this method has many obvious pitfalls, data collision being one.

Data collision [9] is the result of two nodes sending data at the same time on the same channel. A suitable MAC protocol is required to reduce the possibility of this.

\section{a) MAC Protocol Criteria}

The sensor nodes are transmit-only devices. They 'transmit and hope'. It is therefore the responsibility of the system design to ensure the highest possible success rate for sensor data to arrive at its destination, the base station. The first step is to ensure that sensor node data is transferred to the mesh nodes reliably. This requires the implementation of a suitable MAC protocol between sensor nodes and mesh nodes.

In order to choose the appropriate MAC protocol the following issues must be addressed in conjunction with the overall scheme of the system:

- Collision avoidance: The primary task of a MAC protocol is to ensure that data transmissions do not interfere with each other. Some MAC protocols are tolerant of low levels of collision. These normally have a contingency to resend collided data. In order for this to work, a node must contain a radio receiver. As our proposed 
sensor nodes are transmit-only devices, total collision avoidance is necessary.

- Minimum Power Consumption: In wireless sensor networks the main power drain for nodes are the radio components, the transmitter and receiver. Some sensor nodes transmit at regular interval based on a timing scheme. Other nodes respond to events. They transmit data only after an event has occurred. These events may rarely occur, extending the lifetime of the battery considerably. Here, the sensor nodes contain an integrated radio transmitter and microcontroller. Therefore, MAC protocol should ensure that the radio transmitter is use efficiently.

- Scalability: This depends on the application, particularly in relation to the number of nodes required. The MAC protocol should be designed to meet the scalability requirement of the application. Furthermore, it should allow for the ad-hoc deployment of the sensor nodes.

- Data Latency: Latency, in this case, is the time delay from when a sensor node has data to send until the data is received by a mesh node. Acceptable latency depends on the application. There is additional latency associated with sending data from a mesh node to the base station. A MAC protocol should provide an adequate compromise between scalability and latency.

\section{b) Implementation}

As mentioned previously, a suitable MAC layer protocol is required to avoid data collision, thus ensuring successful data transfer for sensor nodes, with acceptable latency. To achieve this, some MAC protocols schedule nodes into sub divisions based on either time (Time Division Multiplexing, TDM) or frequency (Frequency Division Multiplexing, FDM). The rfPIC in the proposed sensor nodes operates on a single frequency channel. FDM requires the use of multiple frequencies and therefore is not an option in this case. TDM is used instead. This requires a dedicated time period to be allocated for each transmitter.

TDM is a multiplexing technique that allows transmission from several sources to access the same communication channel. This technique is often called Time Division Multiple Access (TDMA). TDMA applies division in the temporal domain. Each sensor node is allocated a time slot. This slot is a designated period of time for a device to transfer its data. In this time period all other devices refrain from transmitting, thus guaranteeing an interference free channel.

Time slots may also include guard-bands, which are simply time spaces between slots. They guard against a possible drift in synchronisation and also cater for a tolerance in the timing between different devices. However guard-bands do give rise to additional latency.

Once the width of the time slot and the total number of slots are known, the maximum expected latency can be calculated by multiplying the number of TDM slots by the width of the slot. This formula can also be used to establish trade-offs in the system performance. The system can be designed to meet a short latency requirement by reducing either the time slot width or the number of slots i.e. the number of sensor nodes. Alternatively a large number of sensor nodes can be accommodated by either reducing the slot width or accepting an increase in the maximum latency. Thus, for example, designing the system to have up to 2000 sensor nodes and a maximum acceptable latency of 30 seconds would require a maximum allowable TDM slot width of $15 \mathrm{~ms}$.

The format for the data packet transmitted by the sensor nodes is shown in Figure 5 below. This packet is 32 bits in length. The transmission rate for the sensor node is set to $20 \mathrm{kbit} / \mathrm{s}$, resulting in a bit time of 50us. Hence, the total time required to transmit the entire data packet is $50 \mathrm{us} \times 32=1.6 \mathrm{~ms}$.

\begin{tabular}{|c|c|c|c|}
\hline Bbit Preamble & 12bit ID & 8bit Data & 4bit CS \\
\hline
\end{tabular}

Figure 5: Sensor Node Data Packet

\section{TDM SYNCHRONISATION}

In order to implement the TDM based protocol outlined in the section III, every sensor node must be synchronised at the start of the TDM transmission sequence.

The proposed sensor node incorporates a radio receiver for this purpose. This receiver is capable of receiving a unique broadcast signal that is available to the entire system. This broadcast signal is then used to synchronise the start of the TDM sequence for each sensor node. Once synchronised each node maintains this synchronisation internally using a crystal oscillator. Therefore, reception of the synchronising broadcast signal may only be required every few hours if not days.

In this paper we investigate the use of an existing radio broadcast signal, namely the MSF time signal from Rugby, for the purpose of synchronisation.

\section{a) MSF Time Signal}

The MSF timing signal is a radio broadcast signal of the atomic clock held at the NPL (National Physics Laboratory) Rugby, England. This signal is a modulated $60 \mathrm{kHz}$ carrier wave which transmits a time signal every minute. The time information is sent in a bit stream at a rate of $1 \mathrm{bit} / \mathrm{s}$. Bits are represented by on-off carrier modulation. $A$ ' ' 1 ' and 
' 0 ' bit are represented by switching the carrier wave off for $200 \mathrm{~ms}$ and $100 \mathrm{~ms}$ respectively. The accuracy of the one second interval between bits, transmitted from Rugby, is better than $+/-1 \mathrm{~ms}$ [10].

\section{b) Utilising the MSF Time Signal}

To utilise this ever-changing signal a unique bit pattern must be identified. This could then be used to determine the synchronisation point. It is also necessary that this unique pattern is repeatable at regular intervals. The pattern selected from the broadcast signal is the start of frame (SOF) identifier. Repeated every minute, the SOF signature comprises a $500 \mathrm{~ms}$ low pulse followed by a $500 \mathrm{~ms}$ high pulse, as shown in Figure 6 below.

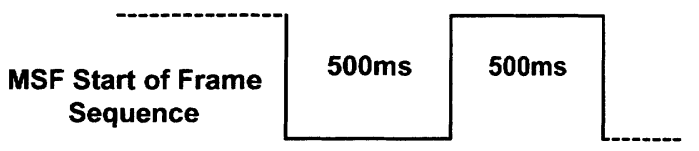

Figure 6: MSF Start of Frame

The sensor nodes contain an EM2S MSF receiver module from Galleon [11] that has a pulse width tolerance of $+/-30 \mathrm{~ms}$. This results in a maximum tolerance between different sensor nodes of $+/$ $60 \mathrm{~ms}$. This tolerance is mainly due to the received MSF signal and not the Galleon module. It was shown previously that a system containing 2000 sensor nodes with a latency of 30 s required a TDM slot width of $15 \mathrm{~ms}$. Therefore, the MSF appears, at first glance, to be a poor choice for synchronisation. However, this is not necessarily the case. The tolerance of $+/-60 \mathrm{~ms}$ is based on the $500 \mathrm{~ms}$ pulse width. A significantly smaller, and more useful, tolerance can be obtained by using consecutive falling edges, as will be shown in the next section.

The proposed synchronisation method is to sample the MSF signal to locate the SOF signature. If the two $500 \mathrm{~ms}$ pulses are both within a certain tolerance, the signal is accepted as a valid SOF. Anything outside this tolerance is discarded. The falling edge of the second pulse is then used to establish the synchronisation point at $t_{1}+t_{2}$, as shown in Figure 7 below. Ideally, $t_{2}=0$, but for practical software related reasons, $t_{2}$ will be set to a few milliseconds. This proposed method is now analysed.

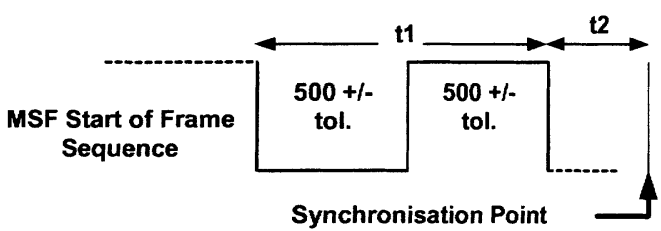

Figure 7: Determining the Synchronisation Point

\section{Preliminary Analysis and Results}

In order to test the success rate of acquiring a MSF SOF signal, a PIC microcontroller from Microchip was connected to the MSF receiver. The PIC was programmed to detect the SOF signal, allowing the following tolerances: $+/-5 \mathrm{~ms},+/-10 \mathrm{~ms},+/-20 \mathrm{~ms}$. The success rate for each was less than $10 \%, 30 \%$, and $90 \%$ respectively. This test was carried out in a good stable reception environment. These results comply with the pulse width tolerance specified by Galleon for their MSF receiver.

Preliminary tests have shown that the MSF receiver is susceptible to interference. Switch mode power supplies in the vicinity of the MSF receiver have a severe detrimental effect on the signal. External interference also corrupts the signal from time to time. The signal however, always recovers from this interference.

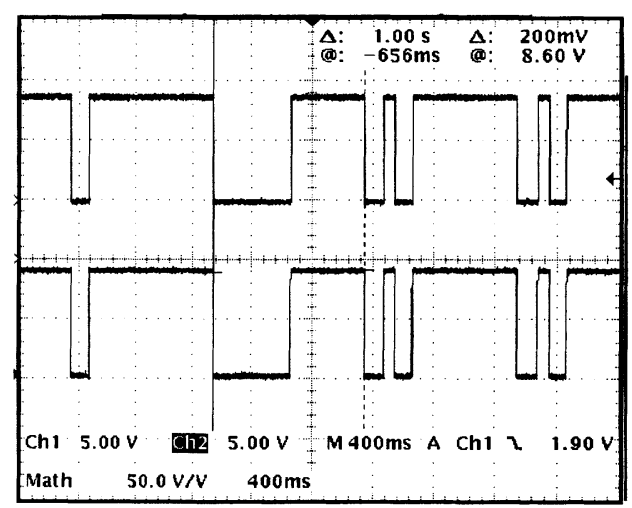

(a)

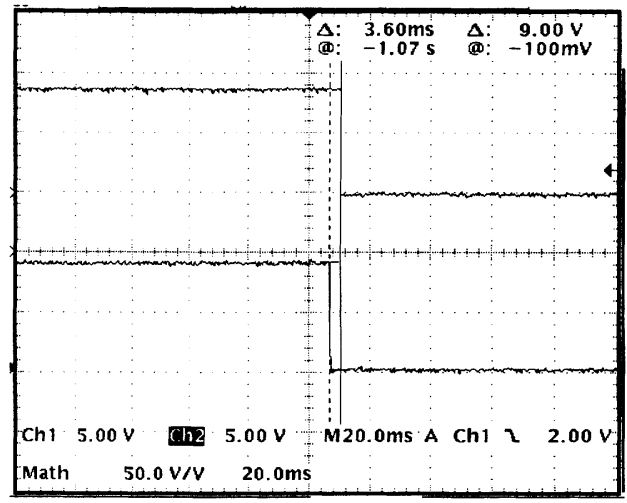

(b)

Figure 8: (a) MSF SOF Signal and (b) Synchronisation Accuracy of SOF.

In order to validate the accuracy of the chosen synchronisation point, the outputs of three MSF receivers were compared, to see how closely aligned these points where. The $500 \mathrm{~ms}$ pulses had to be within a $+/-20 \mathrm{~ms}$ tolerance limit, otherwise the SOF signal was not used. Figure 8 (a) shows the SOF 
signal captured from two of these MSF receivers. It was observed that the accuracy of the falling edge of the 1s SOF signal was within a few milliseconds. Furthermore, this was not dependent on the accuracy of the two individual $500 \mathrm{~ms}$ pulses within the $+/$ $20 \mathrm{~ms}$ tolerance. Figure 8 (b) shows a magnified view of the synchronisation point of both MSF signals. This shows the deviation between the two points to be $3.6 \mathrm{~ms}$.

This test was repeated for continuous samples of the MSF signal. In all instances (to date), a synchronisation accuracy of better than $+/-3 \mathrm{~ms}$ was obtained, thus validating the proposed TDM synchronisation approach. Furthermore, as a point of interest, in approximately $20 \%$ of samples this deviation was less than $1 \mathrm{~ms}$.

It is worth stating that maintaining the pulse width tolerance of $+/-20 \mathrm{~ms}$ has proven to be important. Pulse widths outside this tolerance are normally caused by interference. This interference has resulted in synchronisation errors of up to $20 \mathrm{~ms}$ in the falling edge of the $1 \mathrm{~s}$ SOF signal. It is planned to investigate this issue in more detail.

Until now, the synchronisation tests used a common MSF SOF signature. This signature repeats every minute. It is possible for sensor nodes to synchronise at different times. Therefore a test is required to ensure that different SOF signatures remain within a given tolerance. This was achieved as follows. A PIC was programmed to produce a precise $10 \mathrm{~Hz}$ square wave clock. This clock was used as a reference in determining the accuracy of the MSF SOF signature, from one minute to the next. This was done by referencing the falling edge of the $\mathrm{SOF}$ with an edge of the $10 \mathrm{~Hz}$ clock. It was observed over a period of 10 hours and, while receiving a good MSF signal, it was noted that the accuracy remained within $+/-3 \mathrm{~ms}$.

With a synchronisation accuracy of $+/-3 \mathrm{~ms}$ it is now possible to determine the performance of the system in regards to latency versus number of sensor nodes, as shown in Figure 9. The TDM time slots can be calculated at $7.6 \mathrm{~ms}, 1.6 \mathrm{~ms}$ for the data packet and $6 \mathrm{~ms}$ for the guard band. The scalability of the system is limited by the maximum acceptable latency. With a maximum latency of 30 s, up to 4,000 sensor nodes could be accommodated. For the proposed housing community mesh sensor network, a maximum latency of $30 \mathrm{~s}$ is deemed acceptable.

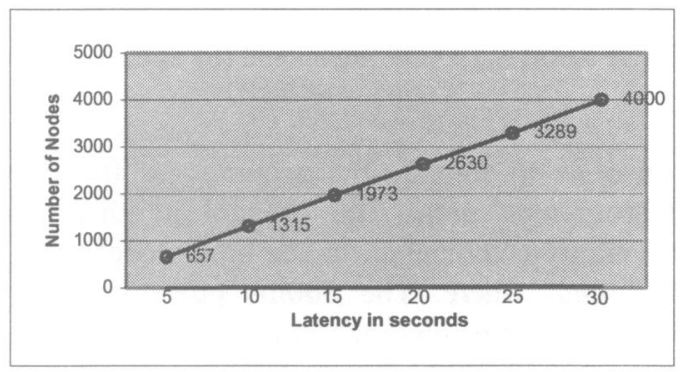

Figure 9: Latency vs. Number of Sensor Nodes

\section{Conclusions and Future Work}

In this paper, we presented a novel design for a mesh sensor network to be used in a housing community. The network uses sensor nodes that are wirelessly connected to the mesh nodes, thus allowing for greater flexibility in sensor deployment A MAC protocol based on TDM was used to ensure a successful transfer of data from the sensors to the mesh nodes. The use of the MSF broadcast for TDM synchronisation was proposed and investigated. It was illustrated that the falling edge of the 1s SOF signature could be readily used for accurate synchronisations within a $+/-3 \mathrm{~ms}$ tolerance limit.

The main focus of future work will be the ongoing research into developing and implementing the system described in this paper. Future testing will also be done on verifying the accuracy of the MSF synchronisation signal over longer periods of time.

\section{References}

[1] P. Cross, "Zeroing in on Zigbee (part 2)," Circuit Cellar Digital Library, Issue 176, March2005.

[2] http://www.bluetooth.com.

[3] http://www.z-wavealliance.org.

[4] http://www.rfid.ie.

[5] IEEE, "Wireless LAN medium access control and physical layer specification," IEEE Std. 802.11-1999 edition, 1999.

[6] T. Culter, "Implementing Zigbee wireless mesh networking", Industrial Automation, July 2005, http://www.rfdesign.com.

[7] W. Ye and J. Heidemann, "Medium Access Control in Wireless Sensor Networks," USC/ISI Technical Report ISI-TR-580, October 2003.

[8] Microchip Corporation, rfPIC12Fxx microcontroller datasheet, http://www.microchip.com.

[9] T. Stathopoulos, R. Kapur, D. Estrin, J. Heidemann, L. Zhang, "Application-Based Collision Avoidance in Wireless Sensor Networks," Proceedings of the $29^{\text {th }}$ IEEE International Conference on Local Computer Networks (LCN'04) 2004.

[10]The National Physics Laboratory, "NPL Time and Frequency Services", June 2005, http://www.npl.co.uk.

[11]OEM Modules, MSF, Galleon Systems, "Receiver Modules and Antenna", http://www.galleon.eu.com, 2003. 